\title{
METRIC PROPERTIES OF TRANSFORMATIONS OF $G$-SPACES
}

BY

\author{
R. K. THOMAS( $\left.{ }^{1}\right)$
}

\begin{abstract}
The measure-preserving transformation $T$ acts on a Lebesgue space $(M, \mathscr{B}, \mu)$ which is also a $G$-space for a compact separable group $G$. It is proved that if the factor-transformation on the space of $G$-orbits has completely positive entropy and a certain condition regarding the relations between the actions of $G$ and $T$ is satisfied, then $T$ weakly mixing implies $T$ has completely positive entropy.
\end{abstract}

Introduction. It is very useful to know that a measure-preserving transformation $T$ has completely positive entropy; if this is the case, then $T$ is mixing of all orders; if, in addition, $T$ is invertible, then $T$ is a Kolmogorov automorphism. An account of all this can be found in Rohlin's survey article [3]. The present paper considers completely positive entropy when the basic measure space $(M, \mathscr{B}, \mu)$ is also a $G$-space for a compact separable group $G$. To be precise, the following theorem is proved:

THEOREM A. Let $T$ be a measure-preserving transformation of a Lebesgue space $(M, \mathscr{B}, \mu)$ which is also a $G$-space for a compact separable group $G$. If $T$ satisfies the following conditions:

(i) $T$ is weakly mixing (has continuous spectrum, see $[4$, p. 39]);

(ii) $T$ o-commutes with $G$-action, i.e. $T \cdot g=\sigma g \cdot T$ for all $g$ in $G$, where $\sigma$ is a group endomorphism of $G$ onto $G$ (see $\S 2$ );

(iii) $T_{\zeta(G)}$ has completely positive entropy (see $\S 1$ ), then $T$ has completely positive entropy.

This theorem "lifts" the property of having completely positive entropy from the factor-transformation $T_{\zeta(G)}$ on the space of $G$-orbits to the transformation $T$ itself. The concepts and notation used in stating the theorem will be considered in more detail in $\S \S 1$ and 2; the proof is given in $\$ \S 3$ and 4 and $\S 5$ considers some applications.

An immediate corollary of Theorem $\mathrm{A}$ is that an ergodic group endomorphism has completely positive entropy (see $\$ 5.1$ ). This result was finally proved by Juzvinskii [1] in 1965, the abelian case having been proved by Rohlin [2] the

Received by the editors March 18, 1970 and, in revised form, October 27, 1970.

AMS 1970 subject classifications. Primary 28A65, 22D40; Secondary 22D05, 22D10, 22E15.

Key words and phrases. Completely positive entropy, $G$-space, $\sigma$-commuting.

$\left.{ }^{1}\right)$ This paper is part of a thesis submitted at the University of Warwick for a Ph.D. degree. Copyright (C) 1971, American Mathematical Society 
previous year. The present result is a generalization; it can be applied to automorphisms of nilmanifolds for example (see §5.3). Also the proof given is more direct. In particular, Juzvinskiî's "Addition Theorem" is not needed. The latter is however an interesting result in its own right and a further paper will be published giving a generalization and modified proof of it.

Much use is made of Rohlin's and Juzvinskiî's work. Their technique of breaking down a group into sequences of factor-groups, on which "elementary" types of endomorphisms are induced, is employed. Some results on the structure of compact groups are quoted directly: proofs of these are given in an appendix as most readers will find the originals rather inaccessible.

I should like to express my gratitude to Dr. W. Parry for his supervision of this work.

1. Entropy theory. It will be assumed that the reader is acquainted with the entropy theory of measure-preserving transformations. The purpose of the brief summary given below is to clarify notation and to state results needed later; all references are to Rohlin's survey article [3].

1.1. Lebesgue spaces and partitions. The fundamental measure space $(M, \mathscr{B}, \mu)$ is a Lebesgue space (normalized so that $\mu(M)=1$ ), i.e. the nonatomic part of $(M, \mathscr{B}, \mu)$ is measure-theoretically point isomorphic to the unit interval with Lebesgue sets and measure. All partitions of $M$ will be assumed to be measurable. A partition $\xi$ generates a subalgebra $\hat{\xi}$ of $\mathscr{B}$ and, conversely, an arbitrary subalgebra $\hat{\xi}$ is generated by a unique partition $\xi$ (uniqueness, equality, etc. are all considered $\bmod 0$ ). The space $M / \xi$ consisting of elements of $\xi$ together with $\hat{\xi}$ and $\mu$ restricted to $\hat{\xi}$ is also a Lebesgue space.

The set of partitions is partially ordered by putting $\xi \leqq \eta$ if $\hat{\xi} \subset \hat{\eta}$; $\varepsilon$ denotes the maximal partition, i.e. the partition of $M$ into distinct points, and $\nu$ denotes the minimal partition, i.e. the trivial partition whose only element is $M$ itself. For a sequence of partitions $\xi_{1}, \xi_{2}, \ldots, \wedge_{n} \xi_{n}$ denotes the largest partition $\xi$ such that $\xi \leqq \xi_{n}$ for all $n$ and $\bigvee_{n} \xi_{n}$ denotes the smallest partition $\eta$ such that $\eta \geqq \xi_{n}$ for all $n$.

1.2. Transformations. For a measure-preserving transformation $T$ and a partition $\xi, T^{-1} \xi$ denotes the partition whose elements are the inverse images of the elements of $\xi$. $\xi$ is said to be $T$-invariant if $T^{-1} \xi \leqq \xi$ and to be completely $T$-invariant if $T^{-1} \xi=\xi$. If $\xi$ is $T$-invariant, then $T$ induces a measure-preserving factortransformation $T_{\xi}$ on $M / \xi . T_{\xi}$ is invertible if and only if $\xi$ is completely $T$-invariant.

For any measure-preserving transformation $T$, there exists a unique maximal completely $T$-invariant partition $\alpha(T)=\bigwedge_{n=0}^{\infty} T^{-n} \varepsilon[3, \S 3.5]$. Clearly, $T_{\alpha}$ is invertible.

1.3. Completely positive entropy. The entropy of a measure-preserving transformation $T$ is denoted by $h(T)[3, \S 9]$. In the set of partitions $\left\{\xi: h\left(T_{\xi}\right)=0\right\}$ there exists a maximal partition $\pi(T)$ known as Pinsker's partition $[3, \S 11.5] . h\left(T_{\eta}^{\prime}\right)=0$ if and only if $\eta \leqq \pi(T) ; \pi(T)$ is completely $T$-invariant and so $\pi(T) \leqq \alpha(T)$; for any integer $n \geqq 1, \pi\left(T^{n}\right)=\pi(T)$, and if $T$ is invertible, $\pi\left(T^{-1}\right)=\pi(T)$. 
$T$ is said to have completely positive entropy if $\pi(T)=\nu$ or, equivalently, if every nontrivial factor-transformation has positive entropy.

If $\xi_{1} \leqq \xi_{2} \leqq \cdots$ is an increasing sequence of $T$-invariant partitions such that $\bigvee_{n} \xi_{n}=\varepsilon$ and $T_{\xi_{n}}$ has completely positive entropy for all $n$, then $T$ has completely positive entropy [3, $\$ 13.4]$.

1.4. Bernoulli automorphisms. An important example of a class of transformations having completely positive entropy is given now. The measure space $M$ is the direct product of a two-way infinite sequence of copies of a Lebesgue space $X$ known as the state space. A point of $M$ is given by a sequence $\left\{x_{n}\right\}_{-\infty}^{\infty}$ and $T$ is defined by $T\left\{x_{n}\right\}=\left\{y_{n}\right\}$, where $y_{n}=x_{n+1}$. This system is known as a Bernoulli automorphism. Of particular interest in the present paper is the case where $X$ is a compact separable group; $M$ is then a compact separable group also and $T$ is a group automorphism. Such a system is known as a Bernoulli group automorphism. All Bernoulli automorphisms have completely positive entropy $[3, \S 13.10]$.

1.5. Automorphisms of tori. A necessary and sufficient condition for an endomorphism $\sigma$ of a compact group to be ergodic is that $U_{i} \sigma^{n}$ should not be equivalent to $U_{i}$ for every irreducible unitary representation $U_{i}$ of the group apart from the constant representation $U_{0}$ [4, p. 54]. In particular, an ergodic group endomorphism has a continuous spectrum.

When $\sigma$ is an automorphism of a finite-dimensional torus, $\sigma$ can be represented as a matrix $A$ with integer entries and determinant \pm 1 . The ergodicity condition becomes: $A$ is ergodic if and only if $A$ has no roots of unity as eigenvalues. Sinar [5] and Arov [6] proved that

$$
h(A)=\log \left|\lambda_{1}\right|+\cdots+\log \left|\lambda_{n}\right|,
$$

where $\lambda_{1}, \ldots, \lambda_{n}$ are the eigenvalues of $A$ of modulus greater than one. So $h(A)$ is zero if and only if all the eigenvalues of $A$ lie on the unit circle. If this is the case, it can be proved that all the eigenvalues are roots of unity. Hence an ergodic group automorphism of a torus has positive entropy [2, \$3.3].

\section{Properties of $G$-spaces.}

2.1. Definition. $(M, \mathscr{B}, \mu)$ is a $G$-space, for a compact separable group $G$, if $G$ acts as a group of invertible measure-preserving transformations of $M$ satisfying the following conditions:

(i) $g \cdot(h \cdot x)=g h \cdot x$ a.e. for all $g, h$ in $G$ and $x$ in $M$;

(ii) $e \cdot x=x$ a.e., where $e$ is the identity of $G$;

(iii) the representation $g \rightarrow U_{g}$ induced on $L^{2}(M)$ by $f(x) \rightarrow f\left(g^{-1} \cdot x\right)=U_{g} f(x)$ is continuous, i.e. for $f, h$ in $L^{2}(M), \int U_{g} f \cdot \bar{h} d \mu$ is a continuous function from $G$ to $C$.

As $g$ is measure-preserving, $U_{g}$ is unitary and so $g \rightarrow U_{g}$ is a continuous unitary representation of $G$.

2.2. Orbit partitions. Suppose that $(M, \mathscr{B}, \mu)$ is a $G$-space as above and that $H$ is a subgroup of $G$. The $H$-orbit partition $\zeta(H)$ of $M$ is defined to be the partition 
generating the algebra

$$
\hat{\zeta}(H)=\{E \in \mathscr{B}: \mu(g E \Delta E)=0 \text { for all } g \text { in } H\}
$$

( $\Delta$ denotes symmetric difference).

Orbit partitions have the following, easily verified, properties:

(i) the continuity of the induced representation of $G$ implies that $\zeta(H)=\zeta(\mathrm{Cl} H)$, where $\mathrm{Cl} H$ is the topological closure of $H$;

(ii) $\zeta(e)=\varepsilon$;

(iii) $H_{1} \supset H_{2}$ implies that $\zeta\left(H_{1}\right) \leqq \zeta\left(H_{2}\right)$;

(iv) if $H_{1} \supset H_{2} \supset \ldots$ is a sequence of closed subgroups of $G$ such that $\bigcap_{n} H_{n}=e$, then the continuity of the induced representation of $G$ implies that $\bigvee_{n} \zeta\left(H_{n}\right)=\varepsilon$;

(v) similarly, if $H_{1} \subset H_{2} \subset \ldots$ is a sequence of subgroups such that cl $\left(\bigcup_{n} H_{n}\right)$ $=G$, then $\bigwedge_{n} \zeta\left(H_{n}\right)=\zeta(G)$.

2.3. $\sigma$-commuting property. The measure-preserving transformation $T$ is said to $\sigma$-commute with $G$-action if $T(g \cdot x)=\sigma(g) \cdot T x$, where $\sigma$ is a group endomorphism of $G$. Clearly, $\zeta(G)$ is $T$-invariant if this happens.

A subgroup $H$ of $G$ is said to be $\sigma$-invariant if $\sigma H \subset H$ and to be completely $\sigma$ invariant if $\sigma H=H$.

If $H$ is $\sigma$-invariant, then $\zeta(H)$ is $T$-invariant.

If $H$ is a $\sigma$-invariant closed normal subgroup of $G$, then $M / \zeta(H)$ is also a $G$-space; in fact, it is a $G / H$-space as $H$ acts trivially on $M / \zeta(H)$. For such an $H, T_{\zeta(H)}$ $\sigma_{G / H}$-commutes with $G / H$-action on $M / \zeta(H)$, where $\sigma_{G / H}$ denotes the endomorphism induced in $G / H$ by $\sigma$.

If $H$ is a completely $\sigma$-invariant closed subgroup of $G$, then $T \sigma_{H}$-commutes with $H$-action, where $\sigma_{H}$ denotes the restriction of $\sigma$ to $H$.

If $T$ is invertible, then $T_{\zeta(G)}$ is invertible also.

2.4. Invariance of $\pi(T)$ under $G$-action. A partition $\xi$ is said to be $G$-invariant if $g \xi=\xi$ for all $g$ in $G . M / \xi$ in this case is also a $G$-space.

If $T \sigma$-commutes with $G$-action and $\sigma(g)=g$ for some $g$ in $G$, then $g \pi(T)$ (and $\left.g^{-1} \pi(T)\right)$ is completely $T$-invariant and $T_{g \pi}\left(T_{g^{-1}}\right)$ is isomorphic to $T_{\pi}$ and so has zero entropy implying that $g \pi \leqq \pi\left(g^{-1} \pi \leqq \pi\right)$ or, equivalently, $\pi \leqq g^{-1} \pi$. Hence $\pi(T)$ must be equal to $g \pi(T)$.

Suppose now that $\sigma$ is densely periodic, i.e. there exists a dense subset $K$ of $G$ such that $\sigma$ is periodic on every element of $K$. For any $g$ in $K, \sigma^{n} g=g$ for some $n$ so that $g \pi(T)=g \pi\left(T^{n}\right)=\pi\left(T^{n}\right)=\pi(T)$. Thus $\pi(T)$ is invariant under all the elements of $K$. The continuity of the induced representation of $G$ implies that $\pi(T)$ is invariant under the whole of $G$.

2.5. Ergodic G-action. $G$-action on $(M, \mathscr{B}, \mu)$ is said to be ergodic if $\zeta(G)=\nu$, i.e. if $\mu(E \Delta g E)=0$ for some set $E$ in $\mathscr{B}$ for all $g$ in $G$ implies that $\mu(E)=0$ or 1 or, equivalently, $U_{g} f=f$ for some $f$ in $L^{2}(M)$ and all $g$ in $G$ implies that $f$ is constant a.e.

3. Reduction of the general case. The purpose of this section is to reduce the general situation of Theorem A to more specific cases. First it is shown that it is 
only necessary to consider cases where $\pi(T)=\varepsilon, T$ is invertible and $\sigma$ is a group automorphism. Then it is shown that it is possible to build up the proof in "steps" using factor groups of $G$. Finally, the work of Rohlin and Juzvinskir is used to show that $G$ can be broken down in such a way that the steps need only be one of four types. The result of all this is that it is sufficient to prove Theorem A for four "elementary" types of group automorphism $\sigma$. The elementary automorphisms are

(i) an automorphism of a finite group,

(ii) an automorphism of a finite-dimensional torus,

(iii) an automorphism of a simple Lie group,

(iv) a Bernoulli group automorphism.

3.1. Assuming that $\pi(T)=\varepsilon$.

LEMMA. If $T \sigma$-commutes with $G$-action on $M, \pi(T)$ is $G$-invariant and $T_{\zeta(G)}$ has completely positive entropy, then $G$ acts ergodically on $M / \pi(T)$.

Proof. Let $\zeta(G, \pi)$ be the $G$-orbit partition of $M / \pi(T)$. By $\S 1.3, h\left(T_{\zeta(G, \pi)}\right)=0$ (since $\zeta(G, \pi) \leqq \pi(T))$. But $\hat{\zeta}(G, \pi) \leqq \hat{\zeta}(G)$, showing that $T_{\zeta(G, \pi)}$ is a factor-transformation of $T_{\zeta(G)}$ and so has completely positive entropy. Hence $\zeta(G, \pi)=\nu$, i.e. $G$ acts ergodically on $M / \pi(T)$.

Suppose now that $T$ satisfies the conditions of Theorem A and that $\pi(T)$ is $G$ invariant. The weakly mixing property of $T$ carries over to $T_{\pi} ; T_{\pi} \sigma$-commutes with $G$-action on $M / \pi(T)$; by the last lemma, $T_{\zeta(G, \pi)}$ is trivial (and so has completely positive entropy), i.e. $T_{\pi}$ satisfies the requirements of Theorem A. It remains to prove that $\pi(T)=\nu$ or, equivalently, $M / \pi(T)$ is trivial (consists of a single atom).

So when it has been established that $\pi(T)$ is $G$-invariant, only $M / \pi(T)$ is considered; $M$ is replaced by $M / \pi(T), T$ by $T_{\pi}$. In other words, in the original formulation of Theorem A, it is assumed that $\pi(T)=\varepsilon$.

3.2. Reduction to invertible transformation and group automorphism. Assume that $T$ satisfies the conditions of Theorem A and let $P$ be any set in $\left(T^{-1} \varepsilon\right)^{\wedge}: P=T^{-1} Q$, where $Q$ is some set in $\mathscr{B}$. Clearly, $g P=T^{-1}(\sigma g \cdot Q)$ and so $g P \in\left(T^{-1} \varepsilon\right)^{\wedge}$. It follows that $T^{-1} \varepsilon$ is $G$-invariant. For $h$ in the closed normal subgroup $\sigma^{-1} e, h P=P$ and so $\sigma^{-1} e$ acts trivially on $M / T^{-1} \varepsilon$. Similarly, $T^{-n} \varepsilon$ is $G$-invariant and $\sigma^{-n} e$ acts trivially on $M / T^{-n} \varepsilon$. Consequently, $\alpha(T)=\bigwedge_{n=0}^{\infty} T^{-n} \varepsilon$ is $G$-invariant and the closed normal subgroup $F=\operatorname{cl}\left(\bigcup_{n=1}^{\infty} \sigma^{-n} e\right)$ acts trivially on $M / \alpha(T)$.

As $\pi(T) \leqq \alpha(T), T$ has completely positive entropy if and only if $T_{\alpha}$ has and so it is sufficient to consider the invertible transformation $T_{\alpha}, \sigma_{G / F}$-commuting with $G / F$-action on $M / \alpha(T) ; \sigma_{G / F}$ being a group automorphism.

3.3. Replacing $T$ and $\sigma$ by $T^{n}$ and $\sigma^{n}$. If $T$ satisfies the conditions of Theorem A, then $T^{n}$ ( $n$ a positive integer) will satisfy these conditions also but with $\sigma^{n}$ in place of $\sigma$. As $\pi\left(T^{n}\right)=\pi(T), T$ and $\sigma$ can always be replaced by $T^{n}$ and $\sigma^{n}$.

Similarly, if $T$ is invertible and $\sigma$ is an automorphism, $T$ and $\sigma$ can be replaced by $T^{-1}$ and $\sigma^{-1}$.

3.4. Proof extension. Assume that $T$ satisfies the conditions of Theorem A. 
(i) Proof by steps. Suppose that $G$ contains a completely $\sigma$-invariant closed normal subgroup $H ; \zeta(H)$ is both $T$ - and $G$-invariant. $T_{\zeta(H)}$ is weakly mixing and $\sigma_{G / H^{-}}$commutes with $G / H$-action on $M / \zeta(H)$. So if $\sigma_{G / H}$ is a type of endomorphism for which Theorem A has been proved, then application gives: $T_{\zeta(H)}$ has completely positive entropy.

$T \sigma_{H}$-commutes with $H$-action on $M$ and $T_{\zeta(H)}$ has completely positive entropy. If now $\sigma_{H}$ is also a type of endomorphism for which Theorem A has been proved, then application gives: $T$ has completely positive entropy.

Thus Theorem A for $\sigma$ follows if Theorem A has been proved for the two "steps" $\sigma_{G / H}$ and $\sigma_{H}$.

This technique can clearly be extended to a finite number of steps: if $G$ contains a sequence $G=G_{0} \supset G_{1} \supset G_{2} \supset \ldots \supset G_{n-1} \supset G_{n}=e$ of completely $\sigma$-invariant closed normal subgroups such that $G_{i}$ is normal in $G_{i-1}$, then Theorem A can be proved for $\sigma$ by proving it for all the steps $\sigma_{G_{i-1} / G_{i}}(i=1,2, \ldots, n)$.

Subsection (ii) shows that the number of steps can be infinite.

(ii) Taking the limit. Suppose that $G$ contains a sequence $G=G_{0} \supset G_{1} \supset G_{2} \supset \ldots$ of $\sigma$-invariant closed subgroups such that $\bigcap_{n} G_{n}=e$. If it is known that $T_{\zeta\left(G_{n}\right)}$ has completely positive entropy for all $n$, then $\$ 2.2(\mathrm{iv})$ and the last sentence of $\S 1.3$ imply that $T$ has completely positive entropy.

Thus Theorem A can be proved for $\sigma$ by proving it for every $\sigma_{G / G_{n}}$. By $§ 3.2$, each step in (i) or (ii) can be assumed to be an automorphism.

For the arbitrary group $G, C$ will denote its connected component of the identity and $Z$ the centre of $C$. Both $C$ and $Z$ are completely invariant under the arbitrary endomorphism $\sigma$. (i) above shows that it is sufficient to prove Theorem $\mathrm{A}$ for $\sigma_{G / C}, \sigma_{C / Z}$ and $\sigma_{Z}$. The remainder of this section is devoted to showing that $G / C$, $C / Z$ and $Z$ all contain sequences of closed normal subgroups such that the endomorphisms induced on factor groups are either elementary automorphisms or are of a type that has previously been considered.

$\sigma_{G / C}$ is considered first; $G / C$ is totally disconnected.

3.5. Totally disconnected groups.

LEMMA (JUZVINSKIǏ $[1, \S 11.5$, SEE APPENDIX A]). If $\rho$ is an automorphism of a compact separable totally disconnected group $H$ and $A$ is an open normal subgroup such that $\bigcap_{-\infty}^{\infty} \rho^{n} A=e$ and $H / A$ is simple, then either

(i) $H$ is finite, or

(ii) $\rho$ is a Bernoulli group automorphism of $H$.

THEOREM. If $\tau$ is an automorphism of a compact separable totally disconnected group $H$, then $H$ contains a sequence $H=H_{0} \supset H_{1} \supset H_{2} \supset \cdots$ of completely $\tau$-invariant closed normal subgroups such that $\bigcap_{n} H_{n}=e$ and either $H_{n} / H_{n+1}$ is finite or $\tau_{H_{n} / H_{n+1}}$ is a Bernoulli group automorphism for all $n$.

Proof. As $H$ is totally disconnected, $H$ contains a sequence $H=E_{0} \supset E_{1} \supset E_{2} \supset \ldots$ of open normal subgroups such that $\bigcap_{n} E_{n}=e . H_{0} / E_{1}$ is finite and so an open 
normal subgroup $F_{1}$ can be found such that $H_{0} \supset F_{1} \supset E_{1}$ and $H_{0} / F_{1}$ is simple. Put $H_{1}=\bigcap_{-\infty}^{\infty} \tau^{n} F_{1} . H_{1}$ is completely $\tau$-invariant and so, applying the lemma, either $H_{0} / H_{1}$ is finite or $\tau_{H_{0} / H_{1}}$ is a Bernoulli group automorphism.

$H_{1} / H_{1} \cap E_{1}$ is finite and so there exists a closed normal subgroup $F_{2}$ such that $H_{1} \supset F_{2} \supset H_{1} \cap E_{1}$ and $H_{1} / F_{2}$ is simple. Put $H_{2}=\bigcap_{-\infty}^{\infty} \tau^{n} F_{2}$ and apply the lemma.

$H_{2} / H_{2} \cap E_{1}$ is finite, etc. This process is continued to obtain $H_{3}, H_{4}$, etc. After a finite number ( $r$ say) of steps, $H_{r} \subset E_{1}$ : when this happens, replace $E_{1}$ by $E_{2}$; then $H_{r} / H_{r} \cap E_{2}$ is finite, etc., to produce $H_{r+1}, H_{r+2}, \ldots$ In due course, $E_{2}$ is replaced by $E_{3}$ and so on. In this way, a sequence $H_{0}, H_{1}, \ldots$ is produced satisfying the requirements of the theorem.

This theorem gives a breakdown of $\sigma_{G / C}$ into a sequence of elementary automorphisms as required. Next, $\sigma_{C / Z}$ is considered; $C / Z$ is a connected group with a trivial centre (see Appendix B).

\subsection{Connected groups with trivial centres.}

THEOREM (JUZVINSKIǏ $[1, \S 4.1$, SEE APPENDIX B]). If $\tau$ is an automorphism of a compact separable connected group with a trivial centre, then $\tau=\tau_{1} \otimes \tau_{2}$, where $\tau_{1}$ is a Bernoulli group automorphism and $\tau_{2}$ is a direct product of automorphisms of semisimple Lie groups with trivial centres.

$\tau_{1}$ is an elementary automorphism and so can be taken as the first step. $\tau_{2}$ is broken down into its summands. Each summand, being an automorphism of a semisimple Lie group, becomes a finite direct product of automorphisms of simple Lie groups (i.e. a direct product of elementary automorphisms) on being raised to some power (this is legitimate by §3.3). So the theorem gives an adequate breakdown of $\sigma_{C / Z}$ for Theorem A for $\sigma_{C / Z}$ to follow from proofs for elementary automorphisms.

Finally, $\sigma_{z}$ is considered.

\subsection{Abelian groups.}

Definition. A (discrete) abelian group $\Gamma$ is said to be finitely generated with respect to an endomorphism $\rho$ of $\Gamma$ if every element of $\Gamma$ can be expressed in the form

$$
\gamma_{1} p_{1}(\rho)+\gamma_{2} p_{2}(\rho)+\cdots+\gamma_{n} p_{n}(\rho)
$$

( $\rho$ operating on the right), where $\gamma_{1}, \gamma_{2}, \ldots, \gamma_{n}$ are fixed elements of $\Gamma$ and $p_{1}, p_{2}, \ldots, p_{n}$ are polynomials with integer coefficients.

First of all, an endomorphism $\rho$ of an abelian group $H$ whose dual group $\Gamma$ is finitely generated with respect to the adjoint endomorphism (also denoted by $\rho$ but written on the right) is considered. It will be shown now that this property is preserved under reduction to invertible transformation and group automorphism (§3.2). This process involves factoring $H$ by a subgroup. In the dual situation, this will mean that $\rho$ is to be restricted to some subgroup $\Omega$ of $\Gamma$. The following lemma gives what is required. 
LemMA (RoHLIN [2, \$3.4, SEE APPENDIX C.1]). If an abelian group $\Gamma$ is finitely generated with respect to an endomorphism $\rho$ and $\Omega$ is a $\rho$-invariant subgroup, then $\Omega$ is finitely generated with respect to the restriction of $\rho$ to $\Omega$.

The following theorem gives a breakdown of $\rho^{-1}$ into elementary automorphisms ( $\rho$ is assumed to be an automorphism and so can be replaced by $\rho^{-1}(\S 3.3)$ ).

TheOREM (Rohlin [2, §4.2, SEE APPENDiX C.2]). If $\rho$ is an automorphism of an abelian group $H$ whose dual group $\Gamma$ is finitely generated with respect to $\rho$, then $H$ contains a sequence $H=H_{0} \supset H_{1} \supset H_{2} \supset \cdots$ of closed subgroups such that $\bigcap_{n} H_{n}=e$, $\rho^{-1} H_{n} \subset H_{n}$ and $H / H_{n}$ is the direct sum of a finite group and a finite-dimensional torus.

Each $\rho_{H / H_{n}}$ in this theorem is a direct product of elementary automorphisms and so Theorem $\mathrm{A}$ for an automorphism satisfying the conditions of the theorem will follow from the proofs for elementary automorphisms on "taking the limit" (§3.4).

Now a general endomorphism $\tau$ of an abelian group $F$ is considered.

TheOREM (Rohlin [2, \$4.3, SEe APPENDix C.3]). If $\tau$ is an endomorphism of a compact separable abelian group $F$, then $F$ contains a sequence $F=F_{0} \supset F_{1} \supset F_{2} \supset \cdots$ of $\tau$-invariant closed subgroups such that $\bigcap_{n} F_{n}=e$ and the dual group of $F / F_{n}$ is finitely generated with respect to $\tau_{F / F_{n}}$ for every $n$.

This theorem implies that $\sigma_{Z}$ can be expressed as the limit of a sequence of factorendomorphisms each one of which satisfies the conditions of the preceding theorem (after reduction to automorphism). The above remarks show how Theorem A follows for such automorphisms. So Theorem A for $\sigma_{z}$ will follow on "taking the limit” (\$3.4).

4. Proof of Theorem A for elementary automorphisms. $T$ is assumed to be invertible and $\sigma$ to be a group automorphism (\$3.2) throughout this section.

4.1. THEOREM. If T satisfies the conditions of Theorem $\mathrm{A}$ with $G$ acting ergodically on $M$, then either

(i) $M$ is trivial, or

(ii) $T$ has Lebesgue spectrum in the orthogonal complement of the invariant subspace of constant functions.

Proof. Representation theory is used; the terminology of [9, Chapter 1] is employed: the compactness of $G$ implies that the representation $U$ induced on $L^{2}(M)$ by $G$-action is directly decomposable into a direct sum of primary representations, i.e. $L^{2}(M)=\sum H_{i}$, where $U$ restricted to $H_{i}$ can be represented as $\lambda_{i} U_{i}$, where $U_{i}$ is an irreducible unitary representation which is unique to within equivalence and $\lambda_{i}$ is its multiplicity (possibly infinite at this stage). This decomposition is unique and $H_{i}$ is orthogonal to $H_{j}$ for $i \neq j\left(U_{i}\right.$ is not equivalent to $U_{j}$ for $\left.i \neq j\right)$. It will be convenient to write $H\left(U_{i}\right)$ for $H_{i}$. 
An orthonormal set of basis vectors

$$
e_{i 11}, e_{i 12}, \ldots, e_{i 1 n}, e_{i 21}, \ldots, e_{i \lambda_{i} 1}, \ldots, e_{i \lambda_{i} n}
$$

( $n$ being the dimension of the representation) is chosen in $H_{i}$ such that

$$
\left(\begin{array}{c}
e_{i j 1}\left(g^{-1} x\right) \\
\vdots \\
e_{i j n}\left(g^{-1} x\right)
\end{array}\right)=U_{i}(g)\left(\begin{array}{c}
e_{i j 1}(x) \\
\vdots \\
e_{i j n}(x)
\end{array}\right)
$$

for $j=1,2, \ldots, \lambda_{i}$. The column of basis functions can be considered as a vector valued function $E_{i j}: M \rightarrow C^{n}$ satisfying the equation $E_{i j}\left(g^{-1} x\right)=U_{i}(g) E_{i j}(x)$. The usual $\boldsymbol{C}^{n}$ scalar product is considered:

$$
\left(E_{i j}\left(g^{-1} x\right), E_{i k}\left(g^{-1} x\right)\right)=\left(U_{i}(g) E_{i j}(x), U_{i}(g) E_{i k}(x)\right)=\left(E_{i j}(x), E_{i k}(x)\right),
$$

i.e. the scalar product is a measurable function which is invariant under $G$-action. The ergodicity of the latter implies that the scalar product is constant a.e.

But

$$
\begin{aligned}
\int_{M}\left(E_{\imath j}, E_{i k}\right) d \mu=\int_{M}\left(e_{i j 1} \bar{e}_{i k 1}+\cdots+e_{i j n} \bar{e}_{i k n}\right) d \mu=0 & \text { if } j \neq k \\
=n & \text { if } j=k .
\end{aligned}
$$

Hence the $E_{i j}$ 's are orthogonal a.e. As there cannot be more than $n$ orthogonal vectors at a point, $\lambda_{i} \leqq n$.

Under $G$-action, the composition of $E_{i j}$ with $T$ behaves as follows:

$$
E_{i j} T\left(g^{-1} x\right)=E_{i j}\left(\sigma\left(g^{-1}\right) T x\right)=U_{i}(\sigma g) E_{i j} T(x) .
$$

So the functions $e_{i 11} T, \ldots, e_{i \lambda_{i} n} T$ are in $H\left(U_{i} \sigma\right)$.

If $U_{i} \sigma^{m}$ were equivalent to $U_{i}$ for some $m$ and some $i \neq 0\left(U_{0}\right.$ denotes the constant representation throughout), then $H\left(U_{i}\right)+H\left(U_{i} \sigma\right)+\cdots+H\left(U_{i} \sigma^{m-1}\right)$ would be a finite-dimensional subspace of $L^{2}(M)$ which remains invariant under $T$; $T$ would then have a nonconstant eigenfunction contradicting the assumption of continuous spectrum.

Thus either

(i) $U=U_{0}$ implying that $M$ is trivial or

(ii) $U_{i} \sigma^{m}$ is not equivalent to $U_{i}(i \neq 0)$ for any $m$, in which case, basis elements $\left\{f_{i, j}\right\}$ can be chosen in $L^{2}(M)$ such that $f_{i, j} T=f_{i, j+1}$, i.e. $T$ has Lebesgue spectrum in the complement of $H\left(U_{0}\right)$.

4.2. Proof of Theorem A for a finite group $G$. Any automorphism $\sigma$ is periodic in this case. So $\pi(T)$ is $G$-invariant $(\$ 2.4)$ and it can be assumed that $\pi(T)=\varepsilon(G$ acts ergodically: $\S 2.5)$. Theorem 3.1 is applied: $U_{i} \sigma^{m}$ must be equivalent to $U_{i}$ for some $m$ for all $i$ and so $M$ must be trivial, completing the proof.

4.3. Proof of Theorem A for $\sigma$ an automorphism of a finite-dimensional torus $G$. $\sigma$ is periodic on the roots of the identity and these are dense in $G$. So, as in the 
previous proof, $\pi(T)$ is $G$-invariant and it can be assumed that $\pi(T)=\varepsilon$ ( $G$ acts ergodically on $M$ ). Theorem 3.1 is applied: if (i) applies, the proof is complete and so it is assumed that (ii) applies, i.e. $U \neq U_{0}$ and $U_{i} \sigma^{m}$ is not equivalent to $U_{i}$ for any $m$ for all $i \neq 0$. As $G$ is abelian, all the irreducible representations are one dimensional and so it follows that all the $H_{i}$ 's in the proof of Theorem 3.1 are one dimensional. A vector $e_{i}$ of unit modulus is chosen from each $H_{i}$ to form an orthonormal basis for $L^{2}(M)$. The $G$-invariance of the $C^{1}$ scalar product implies that $\left|e_{i}(x)\right| \equiv 1$. Thus for $e_{i}$ and $e_{j}, e_{i} \cdot \bar{e}_{j}$ is also in $L^{2}(M)$ and

$$
e_{i} \bar{e}_{j}\left(g^{-1} x\right)=U_{i}(g) \cdot \bar{U}_{j}(g) \cdot e_{i} \bar{e}_{j}(x)
$$

(regarding the $U_{i}$ 's simply as complex valued functions on $G$ ). So the $U_{i}$ 's form a group $B$ (under pointwise multiplication) and $e_{i} e_{j}$ is in $H\left(U_{i} U_{j}\right) . B$ is a subgroup of the dual of $G$ and so is the dual of some torus $G / H$, where $H$ is a completely $\sigma$ invariant closed subgroup. $B$ is a free group on a finite number of generators, $U_{1}, U_{2}, \ldots, U_{n}$ say. By $\S 1.5, \sigma_{G / H}$ can be represented as a matrix $A=\left\{a_{i j}\right\}$ :

$$
U_{i} A=U_{1}^{a_{11}} \cdot U_{2}^{a_{2 i}} \cdot \ldots \cdot U_{n}^{a_{n i}}, \quad i=1,2, \ldots, n .
$$

As $e_{i} T$ is in $H\left(U_{i} A\right)$,

$$
e_{i} T=\mu_{i} e_{1}^{a_{1 i}} \cdot e_{2}^{a_{2 i}} \ldots \cdot e_{n^{n i}}^{a_{1}}, \quad i=1, \ldots, n,
$$

where the $\mu_{i}$ 's are constants of unit modulus depending only on the choice of the $e_{i}$ 's. It is shown now that the $\mu_{i}$ 's can be removed. For a set of constants $\lambda_{1}, \lambda_{2}, \ldots, \lambda_{n}$ of unit modulus, $f_{i}$ is put equal to $\bar{\lambda}_{i} e_{i}$ and substituted into the last

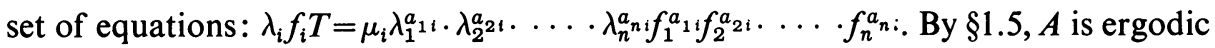
and det $(A-I) \neq 0$ and so the equations $\lambda_{i}=\mu_{i} \lambda_{1}^{a} a_{2 i} \lambda_{2 i}^{a} \cdots \cdot \lambda_{n i}^{a}$ have a solution $\lambda_{i}=\alpha_{i}$ (take log's). For this choice of the $\lambda_{i}$ 's,

$$
f_{i} T=f_{1}^{a_{1 i}} \cdot f_{2}^{a_{2 i}} \ldots \ldots \cdot f_{n}^{a_{n i}}, \quad i=1, \ldots, n .
$$

Let $F$ be the group generated by these $f_{i}$ 's; the elements of $F$ constitute a complete orthonormal basis for $L^{2}(M)$. The map $W: F \rightarrow B$ taking $f_{i}$ to $U_{i}$ is a group isomorphism satisfying $W\left(f_{i} T\right)=\left(W f_{i}\right) A$. Extending first to finite linear combinations and then passing to the limit, $W$ becomes a multiplicative isometry between $L^{2}(M)$ and $L^{2}(G / H)$. The following diagram commutes:

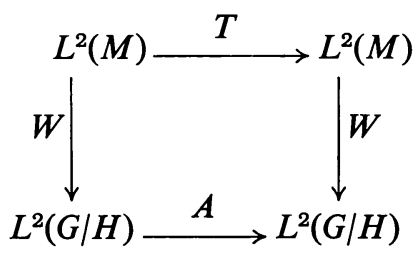

In other words, $T$ and $A$ are equivalent; as $W$ is multiplicative, $T$ and $A$ are also conjugate $[4$, pp. 44 and 45]. Hence $h(T)=h(A)[3, \S 16.3]$ which is positive by $\S 1.5$. This contradicts the assumption $\pi(T)=\varepsilon$ unless $\varepsilon=\nu$. So $M$ must be trivial. 
4.4. Proof of Theorem A for $\sigma$ an automorphism of a simple Lie group G. For a simple Lie group, the subgroup of inner automorphisms is of finite index in the group of all automorphisms [8, p. 193]. So, by replacing $T$ and $\sigma$ by $T^{n}$ and $\sigma^{n}$ if necessary, it can be assumed that $\sigma$ is an inner automorphism: $\sigma(y)=g y g^{-1}$ for some element $g$ in $G$. Putting $T^{\prime}=g^{-1} T, T=g T^{\prime}=T^{\prime} g$. Let $K$ be the closed abelian subgroup of $G$ generated by $g ; K$ is completely $\sigma$-invariant. $G$-action commutes with $T^{\prime}$ and $K$-action commutes with $T$ so that $\pi\left(T^{\prime}\right)$ is $G$-invariant and $\pi(T)$ is $K$-invariant by $\S 2.4$. Clearly $T_{\zeta(K)}=T_{\zeta(K)}^{\prime}$.

It is shown that $T^{\prime}$ has completely positive entropy, from which it will follow that $T_{\zeta(K)}^{\prime}$ and $T_{\zeta(K)}$ have. As in the previous proofs, it is assumed that $\pi\left(T^{\prime}\right)=\varepsilon$. Theorem 3.1 is applied: as $\sigma$ is an inner automorphism, $U_{i} \sigma$ is equivalent to $U_{i}$ for all $i$ and so (i) applies showing that $\pi\left(T^{\prime}\right)=\nu$.

$M$ can be considered now as a $K$-space: $T \sigma_{K}$-commutes with $K$-action on $M$ and $T_{\zeta(K)}$ has completely positive entropy. Completion of the proof depends on a proof of Theorem $\mathrm{A}$ for the automorphism $\sigma_{K}$ of the abelian group $K$; such automorphisms will be dealt with separately in the next section.

4.5. Proof of Theorem A for $\sigma$ a Bernoulli group automorphism of $G$. $\sigma$ is easily seen to be densely periodic and so $\pi(T)$ is $G$-invariant and, as before, it is assumed that $\pi(T)=\varepsilon$ and $\zeta(G)=\nu$.

For $n= \pm 1, \pm 2, \ldots$, let $H_{n}$ be the closed normal subgroup of $G$ consisting of all sequences $\left\{x_{i}\right\}_{-\infty}^{\infty}$ with $x_{i}=e$ for $i \geqq n$. Then, $\sigma H_{n}=H_{n-1} ; \cdots \subset H_{-1} \subset H_{0} \subset H_{1} \subset \cdots$; $\operatorname{cl}\left(\bigcup_{n=1}^{\infty} H_{n}\right)=G$ and $\bigcap_{n=-1}^{-\infty} H_{n}=e . T_{\zeta\left(H_{0}\right)} \sigma_{G / H_{0}}$-commutes with $G / H_{0}$-action on $M / \zeta\left(H_{0}\right)$.

The next step is to replace $\sigma_{G / H_{0}}$ by an automorphism as in $\S 2.6(\mathrm{i})$ :

$$
F=\operatorname{cl}\left(\bigcup_{n=1}^{\infty} \sigma_{G / H_{0}}^{-n} e\right)=\operatorname{cl}\left(\bigcup_{n=1}^{\infty} \sigma^{-n} H_{0}\right)=\operatorname{cl}\left(\bigcup_{n=1}^{\infty} H_{n}\right)=G .
$$

So $G / F=e$, i.e. $G$ acts trivially on $M / \alpha\left(T_{\zeta\left(H_{0}\right)}\right)$. As $G$ acts ergodically, $\alpha\left(T_{\zeta\left(H_{0}\right)}\right)=\nu$. Hence $T_{\zeta\left(H_{0}\right)}$ has completely positive entropy implying that $\zeta\left(H_{0}\right)=\nu$ (since it was assumed that $\pi(T)=\varepsilon)$.

Similarly $\zeta\left(H_{n}\right)=\nu$ for $n=-1,-2, \ldots$ Hence $\varepsilon=\bigvee_{n=-1}^{-\infty} \zeta\left(H_{n}\right)$ (§2.2(iv)) is equal to $\nu$ and the proof of Theorem $\mathrm{A}$ is complete.

\section{Applications of Theorem A.}

5.1. Group endomorphisms. If $M$ is put equal to the group $G$ and $T$ to $\sigma$, then Theorem A states: a weakly mixing group endomorphism has completely positive entropy. An ergodic group endomorphism is known to be mixing ([4, p. 54$]$ and $[10, \S 6])$ and so it follows that an ergodic group endomorphism of a compact separable group has completely positive entropy. This is Juzvinskir's result [1] mentioned in the introduction.

Theorem A can be applied to affine transformations in a similar sort of way to give: a weakly mixing affine group transformation has completely positive entropy. 
5.2. Skew-products. More generally, Theorem A can be applied to the following type of skew-product transformation: $M$ is taken to be a direct product of an arbitrary Lebesgue space $(X, \mathscr{C}, \nu)$ and a compact separable group $G$ with Borel sets and Haar measure $m$ (the latter is also a Lebesgue space; $\nu(X)=m(G)=1$ ); $T$ is given by $T(x, y)=(S x, \sigma(y) \varphi(x))$, where $S$ is a measure-preserving transformation of $X$ and $\varphi$ is a measurable map from $X$ to $G ; T$ will be said to be a skew-product transformation with base $S$ and fibres of type $\sigma$ ( $\sigma$ being a group endomorphism of $G)$. $G$-action is given by $g \cdot(x, y)=(x, g y)$ and so $T g=\sigma g T$, i.e. $T \sigma$-commutes with $G$-action. $T_{\zeta(G)}$ is isomorphic to $S$, and so if $T$ is weakly mixing and $S$ has completely positive entropy, then Theorem A implies that $T$ has completely positive entropy.

5.3. Nilmanifolds. Suppose that $T^{\prime}$ is a group automorphism of a connected and simply connected nilpotent Lie group $N$ (lower central series: $N=N_{0} \supset N_{1} \supset N_{2}$ $\supset \ldots \supset N_{k-1} \supset N_{k}=e$ ) which takes a uniform discrete subgroup $D$ onto itself; the (left) coset space $N / D$ is a compact manifold known as a nilmanifold. $T^{\prime}$ induces a measure-preserving transformation $T$ on $N / D$ known as an "automorphism". This type of transformation has been studied by W. Parry [7] who proved that an ergodic automorphism of a nilmanifold has completely positive entropy. This can be done as follows.

Let $T_{r}$ be the transformation induced on $N / N_{r} D$ and let $\sigma_{r}$ be the transformation induced on $N_{r-1} D / N_{r} D . N_{r-1} D / N_{r} D$ is a torus and $\sigma_{r}$ is a group automorphism of it. The ergodicity condition turns out to be: $T$ is ergodic if and only if $T_{1}$ ("the torus part") is ergodic. Assume now that $T$ is ergodic; $T$ is weakly mixing since the torus part of $T \otimes T$ is $T_{1} \otimes T_{1}$, i.e. the cartesian square of $T$ is ergodic [4, p. 39]. $T_{1}=\sigma_{1}$ is just an ergodic automorphism of a torus and so has completely positive entropy. $T_{2}$ can be regarded as being a skew-product transformation with base $T_{1}$ and fibres of type $\sigma_{2}$ and so has completely positive entropy by $\S 5.2 . T_{3}$ can be regarded as a skew-product transformation with base $T_{2}$ and fibres of type $\sigma_{3}$ etc. So $T_{r}$ has completely positive entropy for $r=1,2, \ldots, k$; in particular, $T_{k}=T$ has completely positive entropy.

Appendix A (Juzvinskii [1, \$11.5]). If $\rho$ is an automorphism of a compact separable totally disconnected group $H$ and $A$ is an open normal subgroup such that $\bigcap_{-\infty}^{\infty} \rho^{n} A=e$ and $H / A$ is simple, then either

(i) $H$ is finite, or

(ii) $\rho$ is a Bernoulli group automorphism of $H$.

Proof (modified slightly). Let $F=H \mid A$ and $\hat{H}=\bigotimes_{-\infty}^{\infty} F_{i}$, where each $F_{i}$ is isomorphic to $F$. Let $\hat{\rho}$ be the Bernoulli group automorphism of $H$ and $p: H \rightarrow F$ be the natural projection with respect to $A$. A map $Q: H \rightarrow H$ is constructed:

$$
Q h=\left\{p\left(\rho^{-i} h\right)\right\}_{-\infty}^{\infty} ;
$$

clearly, $Q \rho=\hat{\rho} Q . Q$ is clearly $1-1$; if $Q$ is onto, then $\rho$ is isomorphic to $\hat{\rho}$ as group automorphisms. Suppose that $Q(H)$ is a proper subgroup of $H$, i.e. there exists a 
sequence $\left\{x_{i}\right\}_{-\infty}^{\infty}$ of elements in $F$ such that $\bigcap_{-\infty}^{\infty} \rho^{i}\left(p^{-1} x_{i}\right)$ is empty. Compactness implies that a finite intersection is empty. Suppose that $\bigcap_{a}^{b}$ is empty but that $\bigcap_{a+1}^{b}$ is not. Then applying $\rho^{-a}$ and multiplying by $x_{a}^{-1}$, this becomes $A \cap D$ is empty, where $D$ is a coset of the open normal subgroup $E=\bigcap_{1}^{b-a} \rho^{i} A$. Thus $A E \neq H$ and so the simplicity of $H / A$ implies that $E \subset A$. Hence $\rho E=E$ and so $E \subset \bigcap_{-\infty}^{\infty} \rho^{n} A=e$. This implies that $e$ is open and consequently that $G$ is finite.

\section{Appendix B (Juzvinskiî's results on compact connected groups).}

LEMMA [1, \$3.3]. If $C$ is a compact connected separable group and $Z$ is its centre, then the factor group $C / Z$ is the direct product of algebraically simple Lie groups. In particular, $C / Z$ has trivial centre.

Proof. André Weil in L'integration dans les groupes topologiques et ses applications proved that $C$ is of the form $(A \otimes B) / N$, where $A$ is a connected abelian group isomorphic to a subgroup of $Z, B$ is a direct product of simple Lie groups and $N$ is a subgroup of the centre of $A \otimes B$. The centre of a direct product is the direct product of the centres of the factors and so $Z=\left(A \otimes Z^{\prime}\right) / N$, where $Z^{\prime}$ is the centre of $B$. So $C / Z$ is isomorphic to $(A \otimes B) /\left(A \otimes Z^{\prime}\right)$ which is isomorphic to $B / Z^{\prime}$. A compact simple Lie group, when factored by its centre, is algebraically simple and so $B / Z^{\prime}$ is a direct product of such groups. Note that separability implies that the direct product is countable.

Note. It is well known in algebra that if a group is the direct product of two nonabelian simple groups, then the only nontrivial normal subgroups are the two factors.

THEOREM [1, \$4.1]. If $\tau$ is an automorphism of a compact separable group $G$ whose centre is trivial, then $\tau=\tau_{1} \otimes \tau_{2}$, where $\tau_{1}$ is a Bernoulli group automorphism of $G_{1}$ and $\tau_{2}$ is a direct product of automorphisms of $\tau$-invariant semisimple Lie groups; $G=G_{1} \otimes G_{2}$.

Proof. By the lemma, $G$ is a countable direct product of algebraically simple Lie groups: $G=\bigotimes_{n} L_{n}$. Let $p_{i}$ denote the projection of $G$ onto $L_{i}$ and $p_{i j}$ the projection onto $L_{i} \otimes L_{j}$. Consider $\tau L_{1}$; this must be a simple normal subgroup of $G$. $p_{i}\left(\tau L_{1}\right)$ can only be $e$ or $L_{i}$. For some $a, p_{a}\left(\tau L_{1}\right)$ must equal $L_{a}$. Suppose also that $p_{b}\left(\tau L_{1}\right)$ $=L_{b}$. Then $p_{a b}\left(\tau L_{1}\right)$ is a normal subgroup of $L_{a} \otimes L_{b}$ and so must equal $L_{a} \otimes L_{b}$ (by the note) contradicting the simplicity of $\tau L_{1}$. So $\tau$ can only permute the $L_{i}$ 's.

$G$ has been shown to be the direct product of $L_{i}$ cycles (under $\tau$ ): $G_{1}$ is put equal to the direct product of all the infinite cycles and $G_{2}$ to the direct product of all the finite cycles. The group of states for $\tau_{1}$ is taken to be the direct product of a simple factor from each infinite cycle.

\section{Appendix C (Rohlin's results on abelian groups).}

COROLlARY 1 [2, §3.4]. If an abelian group $\Gamma$ is finitely generated with respect to an endomorphism $\rho$ and $\Omega$ is a $\rho$-invariant subgroup, then $\Omega$ is finitely generated with respect to $\rho$ restricted to $\Omega$. 
Proof. Every element of $\Gamma$ is of the form

$$
\gamma_{1} p_{1}(\rho)+\gamma_{2} p_{2}(\rho)+\cdots+\gamma_{n} p_{n}(\rho)
$$

(see \$4.4). The result is a special case of a well-known theorem in algebra: if the ring $R$ is Noetherian, then any $R$-module with a finite number of generators is a Noetherian module. In the case given, $R$ is the ring of polynomials with integer coefficients; $R$ is Noetherian by a theorem of Hilbert.

Reference. Van der Waerden, Modern algebra.

COROllary 2 [2, \$4.2]. If $\rho$ is an automorphism of an abelian group $H$ whose dual group $\Gamma$ is finitely generated with respect to $\rho$, then $H$ contains a sequence $H=H_{0} \supset H_{1} \supset H_{2} \supset \cdots$ of closed subgroups such that $\bigcap_{n} H_{n}=e, \rho^{-1} H_{n} \subset H_{n}$ and $H / H_{n}$ is the direct sum of a finite group and a finite-dimensional torus.

Proof. Every element of $\Gamma$ is of the form $\gamma_{1} p_{1}(\rho)+\gamma_{2} p_{2}(\rho)+\cdots+\gamma_{m} p_{m}(\rho)$ (see $\$ 4.4$ ). Let $\Omega_{n}$ be the subgroup of $\Gamma$ obtained by limiting the degrees of the polynomials to $n$. For all $n$ greater than or equal to some $N, \Omega_{n}$ will contain $\gamma_{1} \rho^{-1}, \gamma_{2} \rho^{-1}, \ldots, \gamma_{m} \rho^{-1}$ and so will be invariant under $\rho^{-1}$. The annihilator $F_{n}$ of $\Omega_{n}$ is invariant under $\rho^{-1}$ for $n \geqq N$ and, as $\Omega_{n}$ is finitely generated, $H / F_{n}$ is a direct product of a finite group and a finite-dimensional torus. As $\bigcup_{n} \Omega_{n}=\Gamma$, $\bigcap_{n} F_{n}=e$. So $H_{1}$ is put equal to $F_{N}, H_{2}$ to $F_{N+1}$ etc.

COROLlARY 3 [2, \$4.3]. If $\tau$ is an endomorphism of a compact separable abelian group $F$, then $F$ contains a sequence $F=F_{0} \supset F_{1} \supset F_{2} \supset \ldots$ of $\tau$-invariant closed subgroups such that $\bigcap_{n} F_{n}=e$ and the dual group of $F / F_{n}$ is finitely generated with respect to $\tau_{H / H_{n}}$ for every $n$.

Proof. As $F$ is separable, its dual group $\Gamma$ is countable; so the elements of $\Gamma$ can be indexed $\gamma_{1}, \gamma_{2}, \ldots$ Let $\Omega_{n}$ be the smallest subgroup of $\Gamma$ containing $\gamma_{1}, \gamma_{2}, \ldots, \gamma_{n}$ which is invariant under $\tau$ and let $F_{n}$ be the annihilator of $\Omega_{n}$. $\bigcup_{n} \Omega_{n}=\Gamma$ implying that $\bigcap_{n} F_{n}=e$ and so these $F_{i}$ 's satisfy the given conditions.

\section{REFERENCES}

1. S. A. Juzvinskiir, Metric properties of endomorphisms of compact groups, Izv. Akad. Nauk SSSR Ser. Mat. 29 (1965), 1295-1328; English transl., Amer. Math. Soc. Transl. (2) 66 (1968), 63-98. MR 33 \#2798.

2. V. A. Rohlin, Metric properties of endomorphisms of compact commutative groups, Izv. Akad. Nauk SSSR Ser. Mat. 28 (1964), 867-874; English transl., Amer. Math. Soc. Transl. (2) 64 (1967), 244-252. MR 29 \#5955.

3. - Lectures on the entropy theory of measure preserving transformations with invariant measure, Uspehi Mat. Nauk 22 (1967), no. 5 (137), 3-56=Russian Math. Surveys 22 (1967), no. 5, 1-52. MR 36 \#349.

4. P. R. Halmos, Lectures on ergodic theory, Publ. Math. Soc. Japan, no. 3, Math. Soc. Japan, Tokyo, 1956. MR 20 \#3958.

5. Ja. G. Sinail, On the concept of entropy for a dynamic system, Dokl. Akad. Nauk SSSR 124 (1959), 768-771. (Russian) MR 21 \#2036a. 
6. D. Z. Arov, Calculation of entropy for a class of group endomorphisms, Zap. Meh.-Mat. Fak. Har'kov. Gos. Univ. i Har'kov. Mat. Obšč. (4) 30 (1964), 48-69. (Russian) MR 35 \#4368.

7. W. Parry, Ergodic properties of affine transformations and flows on nilmanifolds, Amer. J. Math. 91 (1969), 757-771.

8. J. Tits, Liesche Gruppen und Algebren, Mathematischen Institut, Bonn, 1965.

9. G. W. Mackey, The theory of group representations, Lecture Notes (Summer, 1955), Department of Mathematics, University of Chicago, Chicago, Ill., 1955. MR 19, 117.

10. I. Kaplansky, Groups with representations of bounded degree, Canad. J. Math. 1 (1949), 105-112. MR 10, 428.

\section{Birkbeck College,}

UNIVERSITY OF LONDON, LONDON, ENGLAND 